\title{
Ultrasonically sculpted tunable relay lenses for imaging and photo manipulation through turbid media (Conference Presentation) (Withdrawal Notice)
}

Matteo Giuseppe Scopelliti, Daniele Busacchio, Maysamreza Chamanzar, Yasin Karimi

Matteo Giuseppe Scopelliti, Daniele Busacchio, Maysamreza Chamanzar, Yasin Karimi, "Ultrasonically sculpted tunable relay lenses for imaging and photo manipulation through turbid media (Conference Presentation) (Withdrawal Notice)," Proc. SPIE 10878, Photons Plus Ultrasound: Imaging and Sensing 2019, 108782W (19 July 2019); doi: 10.1117/12.2510480

SPIE. Event: SPIE BiOS, 2019, San Francisco, California, United States 


\section{Ultrasonically sculpted tunable relay lenses for imaging and photo manipulation through turbid media (Conference Presentation) (Withdrawal Notice)}

Matteo Giuseppe Scopelliti, ${ }^{1}$ Daniele Busacchio, ${ }^{2}$ Maysamreza Chamanzar, ${ }^{1}$ Yasin Karimi ${ }^{1}$

${ }^{1}$ Carnegie Mellon Univ. (United States)

${ }^{2}$ Politecnico di Torino (Italy)

Proc. SPIE 10878, 108782W (2019)

Online Publication Date: 4 March 2019

Withdrawn from Publication: 19 July 2019

Conference Date: 3 February-6 February 2019

Conference Location: San Francisco, California, United States

Conference Title: Photons Plus Ultrasound: Imaging and Sensing 2019

Conference Chairs: Alexander A. Oraevsky, Lihong V. Wang

Publisher's Note: This video, originally published on 4 March 2019, was withdrawn per author request.

Photons Plus Ultrasound: Imaging and Sensing 2019, edited by Alexander A. Oraevsky, Lihong V. Wang, Proc. of SPIE Vol. 10878, 108782W · C 2019 SPIE · CCC code: 1605-7422/19/\$18 · doi: 10.1117/12.2510480 